\title{
REMOCIÓN DE CROMO Y DEMANDA QUÍMICA DE OXÍGENO DE AGUAS RESIDUALES DE CURTIEMBRE POR ELECTROCOAGULACIÓN
}

\author{
Roddy Mayta ${ }^{\mathrm{a}}$, Jhony Mayta $\mathrm{b}^{*}$
}

\begin{abstract}
RESUMEN
Se estudió la remoción de cromo III y demanda química de oxígeno (DQO) de muestras de aguas residuales de curtiduría por electrocoagulación $(\mathrm{EC})$ en un reactor electroquímico de operación discontinua. La influencia de los tiempos (15 a $45 \mathrm{~min})$, voltajes (5 a $10 \mathrm{~V})$, diluciones $(0$ a 2$)$ y sus interacciones en el proceso de EC fueron significativos $(p<0,05)$. Durante el proceso de EC de las muestras se observó incrementos de $\mathrm{pH}$, temperatura y disminuciones de la conductividad. La máxima remoción de cromo III ( 98 \%) y DQO ( 48 \%) fue obtenida con el tratamiento (45 min, $10 \mathrm{~V}$ y dilución de 2). Asimismo, los resultados experimentales permitieron desarrollar modelos matemáticos para la remoción de cromo III y DQO, respectivamente.
\end{abstract}

Palabras clave: Electrocoagulación, cromo trivalente, demanda química de oxígeno.

\section{REMOVAL OF CHROMIUM AND CHEMICAL OXYGEN DEMAND OF TANNERY WASTEWATER BY ELECTROCOAGULATION}

\begin{abstract}
Removal of trivalent chromium and chemical oxygen demand (DQO) of tannery wastewater samples by electrocoagulation (EC) from discontinuous operation electrochemical reactor were studied. The influence of times (15 to $45 \mathrm{~min}$ ), voltages ( 5 to $10 \mathrm{~V}$ ), dilutions ( 0 to 2 ) and their interactions in EC process were significant $(\mathrm{p}<0.05)$. Increases in $\mathrm{pH}$, temperature, and decreases of conductivity of the samples during the EC process were observed. The maximum removal of trivalent chromium $(\sim 98 \%)$ and DQO ( 48 \%) was obtained with the treatment (45 min, $10 \mathrm{~V}$ and dilution of 2). Likewise, the experimental results allowed to develop mathematical models for the removal of trivalent chromium and DQO, respectively.
\end{abstract}

Key words: Electrocoagulation, trivalent chromium, chemical demand oxygen.

\footnotetext{
a Facultad de Ingeniería Química, Universidad Nacional del Altiplano Puno (UNAP), Avenida del Ejército s/n, Puno, Perú.

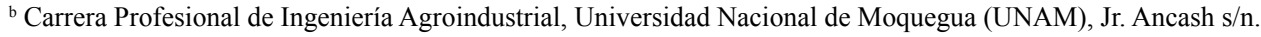
Moquegua, Perú, jhonymayta@hotmail.es
} 


\section{INTRODUCCIÓN}

Los derivados del cromo trivalente se utilizan extensamente como curtientes en el tratamiento del cuero. La industria del cuero, en particular, genera gran cantidad de aguas de curtido y restos de pieles de animales. El sulfato básico de cromo es más utilizado en la curtición de pieles de animales (Golder, Samanta \& Ray, 2007). Las aguas residuales de curtiduría contienen alrededor de 30-35\% de la sal de curtido inicial (Cassano, Molinari, Romano \& Drioli, 2001).

La toxicidad crónica del cromo trivalente (cromo III) es menor que el cromo hexavalente (cromo VI) (USEPA, 1980). Sin embargo, las descargas de efluentes con cromo III en cuerpos receptores con menor dilución de agua pueden provocar una toxicidad por cromo. Por ello, en la mayoría de países del mundo restringieron su vertido a aguas superficiales con contenido de cromo III de 1-2 mg/L (Kimbrough, Cohen, Winer, Creelman \& Mabuni, 1999). Algunos efectos del cromo VI en la salud del ser humano son: alergias en la piel, irritaciones y sangrado de la nariz, erupciones cutáneas, malestar estomacal, alteración del material genético, entre otros. Las intoxicaciones crónicas pueden producir mutaciones en el tracto gastrointestinal y acumulaciones en el hígado, el riñón, la glándula tiroidea y la medula ósea (Costa \& Klein, 2006).

La EC es un proceso electroquímico utilizado en el tratamiento de aguas residuales. Esta técnica presenta múltiples ventajas en comparación a los sistemas biológicos y/o químicos. En el proceso de EC, el coagulante es generado in situ por la reacción de oxidación de un ánodo. Las especies cargadas o metales pesados pueden ser removidos del efluente por la reacción entre iones con carga opuesta o debido a la formación de flóculos de hidróxidos metálicos (Mollah, 2001). Posteriormente, los contaminantes forman componentes hidrofóbicos que se precipitan o flotan, facilitando su remoción por algún método de separación secundario (Petterson, 1985).

La demanda química de oxígeno (DQO) es un análisis no específico pero muy útil para estimar los requerimientos de oxígeno de los vertidos industriales, en los cuales la demanda bioquímica de oxígeno (DBO) no es demasiado eficaz debido a la presencia de tóxicos u otras sustancias inhibidoras (Mancy and Wever, 1971). Las altas concentraciones de DQO en aguas residuales pueden provocar la desoxigenación de las aguas con las que entra en contacto. Este proceso puede afectar el requerimiento de $\mathrm{O}_{2}$ de los organismos acuáticos.

Las sales de cromo utilizadas en la curtiduría del Centro de Investigación de la Facultad de Ingeniería Química de la UNAP contienen cromo III. Este compuesto no es absorbido completamente por la piel durante el proceso de curtición y son eliminados junto con las aguas residuales del proceso sin ningún tratamiento, representando un peligro potencial de intoxicación. Por lo tanto, el objetivo de la presente investigación fue estudiar el efecto del proceso de electrocoagulación por lote en la remoción de cromo III y DQO de las aguas residuales de curtiduría. 


\section{METODOLOGÍA}

\section{Toma de muestras}

Las muestras de aguas residuales del proceso de curtido de pieles de ovino fueron provenientes del Centro de Investigación y Producción de la Facultad de Ingeniería Química - UNAP. Se obtuvo 11 muestras de $15 \mathrm{~L}$ en recipientes de plástico rotulados y de uso exclusivo.

\section{Proceso de electrocoagulación}

El proceso de EC se realizó en batch en un reactor electroquímico provisto de una fuente de poder de $14 \mathrm{~V}$ y 9 A (figura 1). El material de la celda fue de acrílico y tuvo una capacidad de $1,3 \mathrm{~L}$ y $7 \times 7 \mathrm{~cm}^{2}$ de área de reacción. Se utilizó seis electrodos de aluminio (3 ánodos y 3 cátodos) con distancias de $1 \mathrm{~cm}$ entre sí. Antes de realizar el proceso de EC, la celda y los electrodos fueron lavados con una solución de ácido clorhídrico al $4 \%$ durante 10 min y fueron enjuagados con abundante agua potable.

El proceso de EC de las muestras se realizó de acuerdo a la combinación de los niveles de los parámetros de estudio (tabla 1). Las diluciones de las muestras de aguas residuales se realizaron con agua potable. Posterior al proceso de EC las muestras mantuvieron en reposo durante $2 \mathrm{~h}$. El sobrenadante se filtró en papel Watman Nro. 2 y fue envasado y codificado en frascos de vidrio para su análisis correspondiente.

Tabla 1. Niveles de las variables independientes establecidas de acuerdo al diseño factorial $2^{\mathrm{k}}$ con tres puntos centrales.

\begin{tabular}{cccc}
\hline Símbolo & $\begin{array}{c}\text { Parámetro o } \\
\text { variable independiente }\end{array}$ & Nivel bajo (-1) & Nivel alto (+1) \\
\hline A & Tiempo (min) & 15 & 45 \\
B & Voltaje (V) & 5 & 10 \\
C & Dilución (-) & 0 & 2 \\
\hline
\end{tabular}

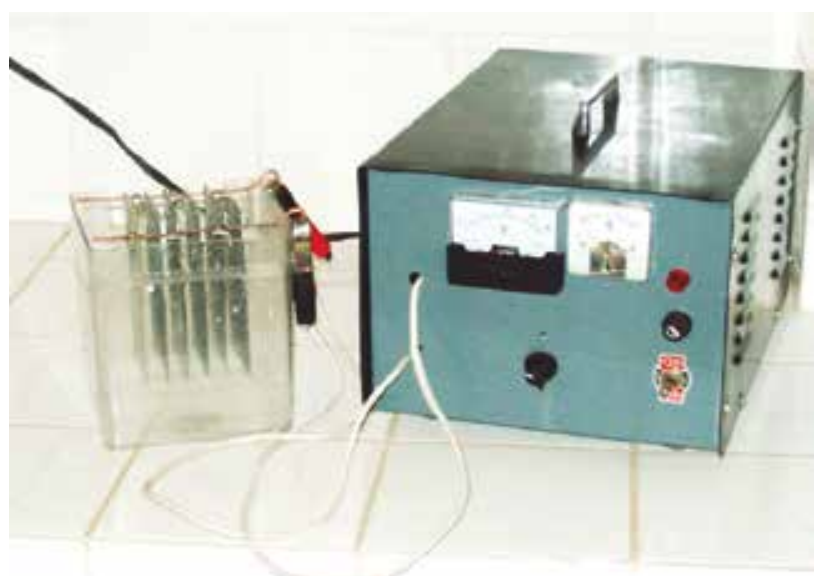

Figura 1. Reactor electroquímico y fuente de poder. 


\section{Métodos de análisis}

Las determinaciones del $\mathrm{pH}$, conductividad $(k)$, temperatura y sales totales disueltas (TDS) se obtuvieron directamente a través de un equipo multiparámetro HI 9828 Hanna. El nitrógeno amoniacal $\left(\mathrm{N}-\mathrm{NH}_{4}\right)$ se determinó por el método Nessler (HACH, 2017). Asimismo, la determinación de DQO se realizó por el método de digestión en reactor $(\mathrm{HACH}, 2014)$ en los laboratorios de la Facultad de Ingeniería Química - UNAP y la Municipalidad Distrital de José Domingo Choquehuanca, Puno, Perú. Las determinaciones de cromo III y cromo total se realizaron de acuerdo al método de absorción atómica por horno de grafito (EPA, 1978) y el método espectrofotométrico propuesto por Castro et al. (1997), respectivamente. Las muestras fueron analizadas en el Laboratorio de Calidad Ambiental de la Universidad Mayor de San Andrés (UMSA) La Paz, Bolivia y de la Facultad de Ingeniería Química - UNAP, Perú.

Las determinaciones de cromo III y DQO se expresaron en porcentajes de remoción utilizando la siguiente ecuación.

$$
\boldsymbol{\eta}=\frac{C_{i}-C_{f}}{C_{i}} \times 100
$$

Dónde: $\boldsymbol{\eta}$ es el porcentaje de remoción, $C_{i}$ es la concentración inicial y $C_{f}$ es la concentración final.

\section{Análisis estadístico}

Se utilizó un diseño factorial $2^{\mathrm{k}}$ (Montgomery, 2013) con tres puntos centrales considerando a los tiempos de residencia (A), voltajes (B) y diluciones de agua (C) (tabla 1). Se realizó tres réplicas del experimento. Los datos obtenidos fueron sometidos a un análisis de varianza (ANOVA) y la determinación de las diferencias significativas entre los tratamientos se realizó por medio de la prueba de medias de Duncan. El nivel de significancia fue determinado a $P<0,05$.

\section{RESULTADOS}

\section{Análisis fisicoquímico de aguas residuales}

Las características fisicoquímicas de las muestras de aguas residuales provenientes del proceso de curtición se muestran en la tabla 2. Valores similares de cromo, $\mathrm{pH}, k$ y sales disueltas en muestras de aguas residuales de curtiembre también fueron observados por Mendoza (2009). 
Tabla 2. Comparativo de los resultados del análisis fisicoquímico de las muestras de agua residual del proceso de curtición y LMP de actividades de curtiembre

\begin{tabular}{lccc}
\hline \multirow{2}{*}{ Parámetros } & Resultados & \multicolumn{2}{c}{ LMP $^{1}$ de efluentes de curtiduría para: } \\
\cline { 3 - 4 } & & Alcantarillado & Aguas superficiales \\
\hline $\mathrm{pH}$ & 4,22 & $6,0-9,0$ & $5,0-8,5$ \\
Temperatura $\left({ }^{\circ} \mathrm{C}\right)$ & 15,00 & 35 & 35 \\
$k\left(\mathrm{mS} \mathrm{cm}^{-1}\right)$ & 36,05 & -- & -- \\
$\mathrm{TDS}\left(\mathrm{mg} \mathrm{L}^{-1}\right)$ & 17,36 & -- & -- \\
$\mathrm{DQO}\left(\mathrm{mg} \mathrm{L}^{-1}\right)$ & 1132 & 1500 & 50 \\
Como III $\left(\mathrm{mg} \mathrm{L}^{-1}\right)$ & 1470 & -- & -- \\
Cromo VI $\left(\mathrm{mg} \mathrm{L}^{-1}\right)$ & -- & 0,4 & 0,2 \\
Cromo total $\left(\mathrm{mg} \mathrm{L}^{-1}\right)$ & 1586 & 0,2 & 0,5 \\
$\mathrm{~N}-\mathrm{NH}_{4}\left(\mathrm{mg} \mathrm{L}^{-1}\right)$ & 64 & 30 & 10 \\
\hline I Decreto Supremo & $\mathrm{N}^{\mathrm{o}} 003$-PRODUCE $(2002)$ & &
\end{tabular}

En general, se obtuvieron menores valores de $\mathrm{pH}$, temperatura y mayores valores de $\mathrm{N}-\mathrm{NH}^{4}$ de las aguas residuales de curtiembre en comparación a los límites máximos permisibles (LMP) de efluentes para alcantarillado de las actividades de curtiembre (DS 003-PRODUCE, 2002). El nivel de DQO de las muestras fueron menores en comparación a los LMP para aguas de alcantarillado, pero resultó mayor para los LMP de aguas superficiales. Los LMP para cromo VI y cromo total (tabla 2) fueron ampliamente superados por los niveles de cromo III de las muestras de aguas residuales del proceso de curtición, representando un peligro para la salud (Costa \& Klein, 2006).

\section{Análisis de muestras tratadas por electrocoagulación.}

Los resultados del análisis $\mathrm{pH}$, conductividad, temperatura, cromo III y DQO de las muestras se observan en la tabla 3. En general, todos los tratamientos presentaron incrementos en el $\mathrm{pH}$ y la temperatura y disminuciones de la conductividad de las muestras durante el proceso de EC. Incrementos de $\mathrm{pH}$ similares también fueron observados por Martínez (2007) en aguas residuales industriales y atribuido a la capacidad amortiguadora del proceso de EC y la formación de iones $\mathrm{OH}^{-}$en el cátodo durante el proceso de reducción de agua. Por otro lado, Arango (2007) también observó incrementos de temperatura durante el proceso de EC y atribuido al desgaste de la película de óxido de aluminio de la superficie del electrodo y también por la liberación de calor en la reacción entre la solución y los electrodos. Finalmente, la disminución de la conductividad en efluentes es debido a la alta concentración de contaminantes. La mayor proximidad entre los iones de la solución inhibe la actividad de la conductividad y pierde su habilidad de transmitir corriente (Lenntech, 2007).

Los tratamientos EC03 y EC04 presentaron menores niveles de cromo III y DQO y, por consiguiente, mayores fueron las tasas de remoción (tabla 2) y visualmente fueron los 
más trasparentes en comparación a las muestras sin ningún tratamiento (figura 2). Golder et al. (2007) observaron una remoción casi completa (99\%) de cromo III utilizando una configuración bipolar a una corriente de $1000 \mathrm{~mA}$ en comparación a la remoción de 81,5 \% para una configuración monopolar. Gilpavas (2008) también logró remover un $\sim 72 \%$ de cromo III y $19 \%$ de DQO en aguas residuales de curtiembre en un reactor con configuración monopolar con electrodos de hierro y aluminio. Uno de los factores que afectaron la solubilidad del cromo III fue la mayor variación del pH obtenido en EC04. Golder et al. (2007) observaron una mínima solubilidad de cromo III en un rango de $\mathrm{pH}$ de 5,8 a 9,3 en disoluciones que no contienen iones cloruro.

Las superficies de respuesta y las ecuaciones optimizadas de remoción de cromo III y DQO se muestran en la figura 3. Asimismo, las interacciones entre el tiempo, voltaje y dilución en la remoción de cromo III produjo un efecto significativo, mientras que en la remoción de DQO, la interacción voltaje y dilución fue significativa (tabla 3). Mayores tasas de remoción de cromo III y DQO se obtuvieron aplicando altos voltajes y mayores diluciones (tabla 2). El efecto del voltaje en la remoción de cromo III podría depender de la corriente y la resistencia del medio (Golder, Samanta \& Ray, 2007; Mollah, 2001) debido a una alta conductividad y baja agitación del medio. Respecto a la dilución, Gilpavas (2008) también logró remover hasta un $\sim 99 \%$ de cromo III y $60 \%$ de DQO en aguas residuales de curtiembre y atribuido a las características y diseño de la celda electrolítica. Las concentraciones elevadas de iones de cromo en la solución dificultan el proceso de remoción (Golder, Samanta \& Ray, 2007; Mollah, 2001). Sin embargo, los tiempos de residencia (15 y $45 \mathrm{~min}$ ) no parecen influir en la mayor remoción de cromo III y DQO del experimento. Estos resultados podrían explicarse por la acumulación de burbujas de $0_{2}$ e $\mathrm{H}_{2}$ en los electrodos a los 10 min del proceso de EC. Mollah (2001) también observó formaciones de placas de óxido que disminuyeron el transporte de masa entre los electrodos y la solución.

a

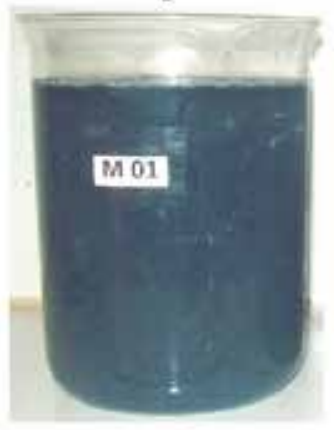

b

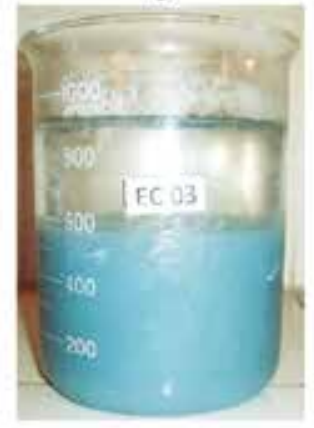

c

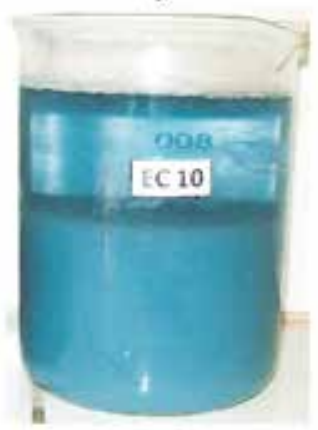

Figura 2. a) muestra inicial del agua residual del proceso de curtición, b y c) aguas residuales tratadas por electrocoagulación. 


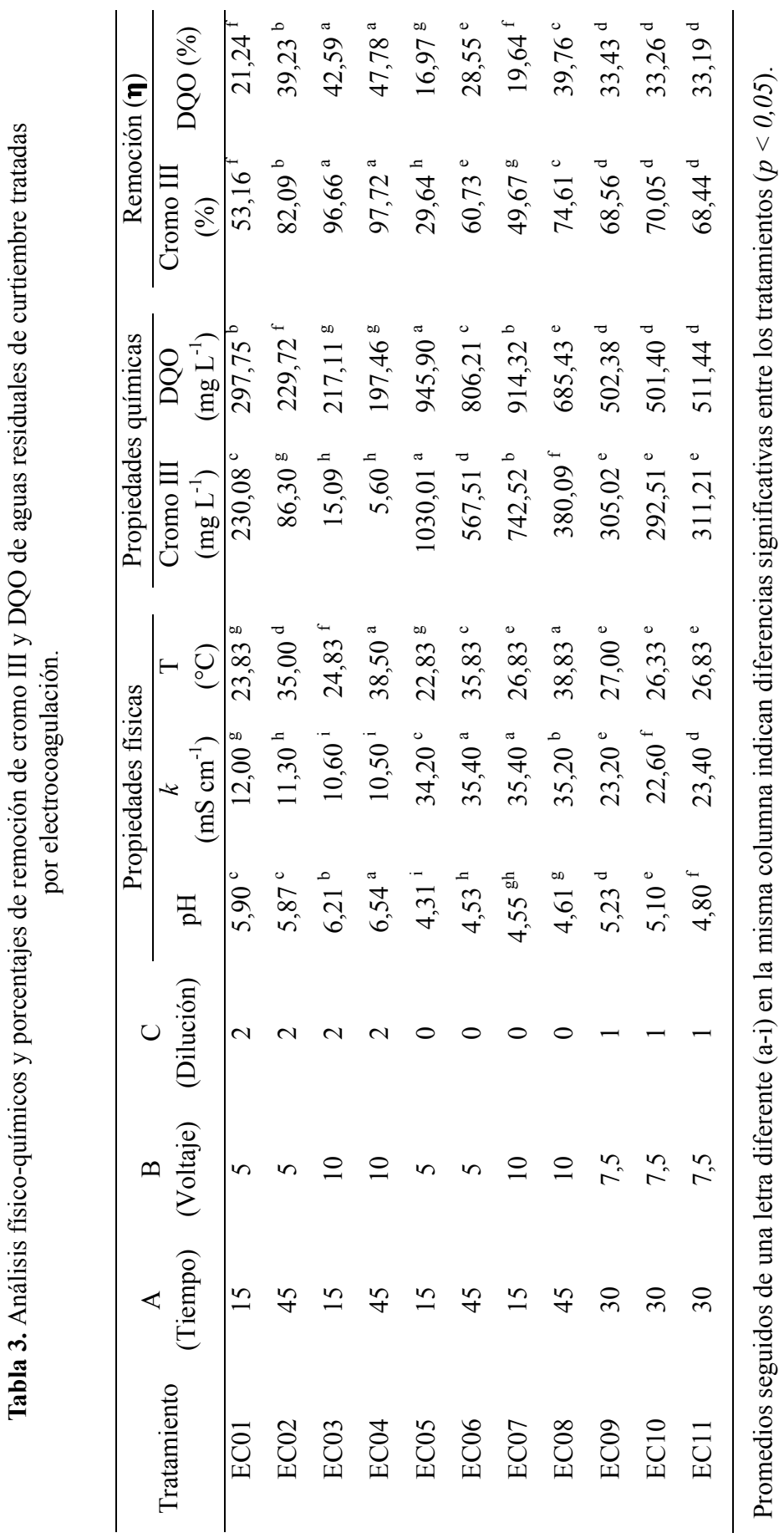


Tabla 4. Cuadrados medios $(\mathrm{CM})$ y probabilidades $(P)$ para remociones de $\mathrm{Cr}^{+3}$ y DQO

\begin{tabular}{|c|c|c|c|c|c|c|}
\hline \multirow{2}{*}{ Factores } & \multicolumn{3}{|c|}{ Remoción de $\mathrm{Cr}^{+3}$} & \multicolumn{3}{|c|}{ Remoción de DQO } \\
\hline & gl & $\mathrm{CM}$ & $P$ & Gl & $\mathrm{CM}$ & $P$ \\
\hline Bloques & 2 & 16,64 & 0,152 & 2 & 9,26 & 0,325 \\
\hline A: Tiempo & 1 & 2773,07 & 0,000 & 1 & 1130,925 & 0,000 \\
\hline B: Voltaje & 1 & 3246,63 & 0,000 & 1 & 718,020 & 0,000 \\
\hline C: Dilución & 1 & 4957,08 & 0,000 & 1 & 792,022 & 0,000 \\
\hline $\mathrm{A} \times \mathrm{B}$ & 1 & 434,52 & 0,000 & 1 & 6,883 & 0,359 \\
\hline $\mathrm{A} \times \mathrm{C}$ & 1 & 253,89 & 0,000 & 1 & 27,118 & 0,076 \\
\hline $\mathrm{B} \times \mathrm{C}$ & 1 & 238,01 & 0,000 & 1 & 95,895 & 0,002 \\
\hline Error & 24 & 8,17 & & 24 & 7,859 & \\
\hline Total & 32 & & & 32 & & \\
\hline
\end{tabular}

a)

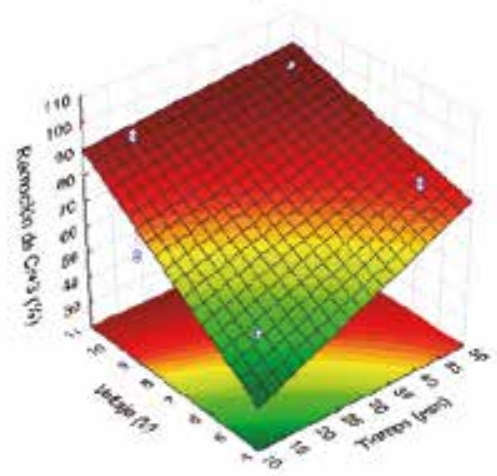

$\eta \operatorname{Cr} I I I(\%)=-2,19+1,36 A+9,32 B$

$-11,42 C-0,11 A B$

$+0,22 A C-1,26 B C$ b)

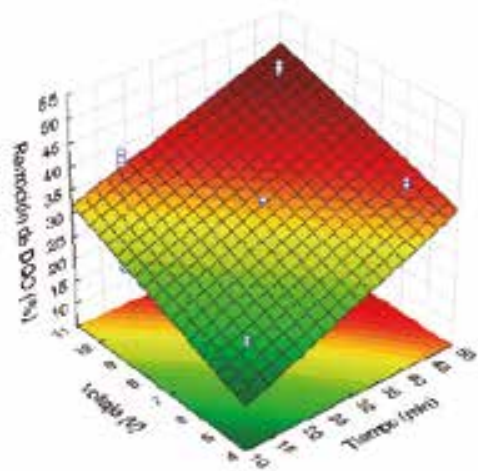

$$
\begin{aligned}
\eta D Q O & (\%)=0,85+0,49 A+3,42 B-1,87 C \\
& -0,01 A B+0,07 A C \\
& -0,80 B C
\end{aligned}
$$

Figura 3. Superficies de respuesta y ecuaciones de remoción de cromo III (a) y DQO (b) 


\section{CONCLUSIONES}

Este estudio muestra que la electrocoagulación de las muestras de aguas residuales de curtiembre permitió remover significativamente el contenido de cromo trivalente y la demanda química de oxígeno, lográndose mejores resultados con mayores diluciones, voltajes y tiempos de residencia.

\section{BIBLIOGRAFÍA}

1. Golder AK, Samanta AN, Ray S. Removal of Cr3+ by Electrocoagulation with Multiple Electrodes: Bipolar and Monopolar Configurations. J Hazard Mater. 2007 Mar; 141(3): 653-661.

2. Cassano A, Molinari R, Romano N, Drioli E. Treatment of aqueous effluents of the leather industry by membrane processes: a review. J Membr Sci. 2001 Jan; 181(1): 111126.

3. USEPA. Ambient Water Quality Criteria for Chromium, EPA-440/5-80-035, NTIS PB85-227478, Office of Water Regulations and Standards, Criteria and Standards. U.S. Environmental Protection. 1980.

4. Kimbrough DE, Cohen Y, Winer AM, Creelman L, Mabuni C. A Critical Assessment of Chromium in the Environment. Crit Rev Environ Sci Technol. 1999; 29(1):1-46.

5. Costa M, Klein CB. Toxicity and Carcinogenicity of Chromium Compounds in Humans. Crit Rev Toxicol. 2006 Feb; 36(2):155-163.

6. Mollah MY, Schennach R, Parga JR, Cocke DL. Electrocoagulation (EC) - science and applications. J Hazard Mater. 2001; 84(1): 29-41.

7. Patterson JW. Industrial wastewater treatment technology. Second edition ed. Stoneham, MA: Butterworth Publishers, Stoneham; 1985.

8. Mancy KH, Weber WJ. Analysis of Industrial Wastewaters. New York: Wiley, NY; 1971.

9. HACH. Hach Company World Headquarters. Nitrogen-Ammonia, Nessler Method/ Method 8038. [Internet]. 2017 [citado 12 set 2017] Disponible en: https://www.hach. com/nitrogen-ammonia-reagent-set-nessler/product-downloads? $\mathrm{id}=7640208228$.

10. HACH. Hach Company World Headquarters. Oxygen Demand, Chemical, Dichromate Method/ Method 8000. [Internet]. 2014 [citado 12 set 2017]. Disponible en: https:// www.hach.com/asset-get.download.jsa?id=7639983816.

11. EPHA. Chromium (AA, Furnace Technique). EPA-NERL: 218.2: Chromium by Graphite Furnace AA. Methods for the Chemical Analysis of Water and Wastes (MCAWW) (EPA/600/4-79/020). [Internet]. 1978 [citado 2 dic 2016]. Disponible en: https://www.nemi.gov/methods/method_summary/5283/.

12. Castro ML, Ikeda K, Suárez D. Metodología para la determinación de Cr (III). Centro Panamericano de Investigación Científica; 1997.

13. Montgomery DC. Design and analysis of experiments. Hoboken: Wiley; 2013.

14. Mendoza B. Tratamiento de aguas residuales procedentes de la planta de curtiembre del Centro de Investigación y Producción de la Facultad de Ingeniería Química. [Tesis de 
pregrado]. Puno: Universidad Nacional del Altiplano; 2009.

15. DS 003-PRODUCE. Aprueban Límites Máximos Permisibles y Valores Referenciales de efluentes para alcantarillado y aguas superficiales para las actividades industriales de cemento, cerveza, curtiembre y papel. Normas legales. Diario Oficial El Peruano. [Internet]. 2002 [citado 5 ago 2011]. Disponible en: http://diariooficial.elperuano.pe/ normas.

16. Martínez F. Tratamiento de aguas residuales mediante electrocoagulación y coagulación convencional. Memoria para optar el grado de Doctor en Ingeniería Química. Ciudad Real: Universidad de Castilla la Mancha; 2007.

17. Arango R. Diseño de una celda de electrocoagulación para el tratamiento de aguas residuales de la inductria lactea. Rev Univ EAFIT. 2007; 43(147): 56-67.

18. Gilpavas E. Aplicación de la electroquímica en el tratamiento de aguas residuales. Medellín- Colombia: Universidad EAFIT; 2008 